\title{
Perceptions of the family physician from adolescents and their caregivers preparing to transition to adult care
}

\author{
Angela Xiao Han ${ }^{1 *}$, Sandy Rosalie Whitehouse ${ }^{2}$, Steve Tsai ${ }^{2}$, Sandy Hwang ${ }^{3}$ and Sally Thorne ${ }^{4}$
}

\begin{abstract}
Background: Adolescents with chronic health conditions and/or disabilities (CHC/D's) often face challenges when transitioning to adult care, which leads to a higher risk of morbidity and mortality. Although it is recommended that establishing the medical home and family physician (FP) attachment prior to transfer will improve health outcomes, there is little evidence or policy surrounding the role of the FP during this transition. This study explores the described use of health services by adolescents with CHC/D's, as well as the adolescent's and caregiver's perceptions of their FP.

Methods: Participants were recruited from the British Columbia Children's Hospital, Canada and a multi-method phased approach was used. In Phase 1, 84 adolescent and caregiver pairs completed questionnaires asking what medical services they accessed for specific health needs. In Phase 2, another cohort of 21 adolescent and caregiver pairs completed the same questionnaires and were interviewed regarding their perception of their FP.

Results: $96 \%(n=81)$ of adolescents with CHC/D's in phase 1 had a FP. Thirty four percent $(n=34)$ of adolescents had not seen their FP in the last 6 months. While adolescents with CHC/D mostly accessed their FP for primary health issues, they frequently also accessed specialists for prescription refills $(50 \%, n=51)$, mental health $(29 \%, n=30)$ and sexual health $(16 \%, n=16)$. While most adolescent/caregiver participants reported positive communication and trust in their FP, some had a poor understanding of the FP's role in coordinating care.

Conclusion: As many adolescents with CHC/D may see their FP infrequently and may not clearly understand their role, opportunities exist for strengthening primary care home attachment as well as adolescent and caregiver literacy around the potential contribution of the FP during and after transfer to adult services. Responsibility for improving care coordination for this population should be ideally shared between FPs and pediatric specialists.
\end{abstract}

Keywords: Adolescent, Chronic illness, Family physician, Medical home, Transition

\section{Background}

An estimated $15-18 \%$ of youth in North America have a chronic health condition and/or disability (CHC/D) and over $98 \%$ of these youth are expected to reach age 20 , thereby requiring transition out of a familiar, family-focused pediatric care system to a dramatically different adult care system where the family physician (FP) is the primary care provider [1]. However, adolescents often feel lost in the adult care system [2]. The adult medicine culture is less attenuated to the developmental complexities of adolescents

\footnotetext{
* Correspondence: axhan@ualberta.ca

${ }^{1}$ University of Alberta, 116 St \& 85 Ave, Edmonton, AB T6G 2R3, Canada Full list of author information is available at the end of the article
}

and focuses on independence, making the transition from an integrated interdisciplinary pediatric system challenging [3]. Lack of engagement with the adult system is associated with lower rates of follow-up appointments and medical compliance $[2,4]$ and a higher risk of mortality and morbidity [5]. While the primary care doctor has a unique opportunity to address primary health care needs and facilitate the transition process through continuity of care $[1,6-8]$, much of the emphasis in the transition literature (which most likely reflects clinical practice) focuses solely on specialist-to-specialist provider transition $[9,10]$.

Young adults have suggested a number of strategies that may facilitate effective transition including peer 
support, mentorship, and having a health care professional who has knowledge and personal understanding of their condition oversee the transfer phase [11]. This concept aligns with the "medical home" model [6]. Implementing a medical home model where health care services are accessible, family centered, continuous, comprehensive, coordinated, and compassionate improves transition preparedness [12, 13]. The patient's medical home can be defined as the place that patients feel most comfortable to discuss their health care, particularly for sensitive topics such as sexual health and mental health. It is a central hub to provide timely and coordinated care by an ongoing team of multidisciplinary caregivers, enabling best possible health outcomes [14]. There is evidence that a "shared care" model that includes patient engagement with specialists and FPs both prior to and after transfer results in better outcomes for adolescents with chronic health conditions such as diabetes [15].

In Canada, the medical home should be established at a FP's practice as they are the first point of contact and primary coordinator of care [14]. However, not all pediatric patients have a FP as some general pediatricians provide on-going primary care in certain provinces. Patients may also access walk-in clinics staffed by different FPs, or emergency departments if they do not have a FP or if their FP is not available. Once a referral is made to a specialist, patients may continue to be followed by that specialist without the involvement of a FP. Although establishing a medical home with a primary care team leads to more effective transition planning, transition protocols often fail to adequately consider the role of the FP in long term management of adolescents following transition [16]. There is little empiric evidence to guide practice or policy surrounding the role of the primary health provider during this transition [1].

The patient-FP relationship is mediated by a variety of factors including the complexity of illness, inclusive management of the patient/family at a tertiary hospital, geography and accessibility to FPs, the perceived role and importance of the FP in continuing care, and the ability to find an FP $[17,18]$. Furthermore, adolescents with $\mathrm{CHC} /$ Ds and their families may choose walk-in clinics, community pediatricians, the internet, an alternative health provider, or a member of their subspecialty team for interim health care management between necessary specialist appointments [19]. Although these may be convenient resources, lack of continuity prevents the establishment of the medical home at the FP's office [20]. Another consideration regarding the patient-FP relationship unique to the transition period is the relationship between the adolescent and their caregiver with respect to health care management and decision-making, such as arranging appointments or seeing the physician alone during appointments. As adolescent patients transfer from pediatric family-centered care to autonomous adult care, the role of their caregiver in care coordination and management transforms. During this period, the adolescent and caregiver can be considered a decision-making unit in transition [21].

This study investigates adolescents with $\mathrm{CHC} / \mathrm{Ds}$ ' described use of health care services and the adolescent and caregiver's perspectives on the FP's role in health care management prior to transfer to adult services. The goal is to inform strategies to improve the continuity of care with FPs through transition and transfer. We used descriptive and qualitative methods to investigate adolescent patients' attachment with their FP by asking if they and their caregivers identified a FP, how often they saw them, and which health services they accessed for specific primary care issues. We also sought to determine the adolescent/caregiver's perceived role of FPs in regular ongoing management and sentiments surrounding trust, knowledge, and overall relationship with their FP.

\section{Methods}

This study was conducted in two phases using mixed methodology at the British Columbia Children's Hospital $(\mathrm{BCCH})$ in Vancouver, Canada. Phase 1 was conducted in 2013 and involved an online questionnaire that included multiple choice and open-ended responses. Phase 2 was conducted in 2014 and included the same questionnaire, with the addition of a qualitative face-to-face interview component that was informed from the open-ended data gathered from phase 1. The University of British Columbia Research Ethics Board approved the study and informed consent was obtained from all participants.

In Phase 1 , a convenience sample $(n=84)$ of adolescent patient/caregiver pairs were invited to participate from the in-patient wards and during subspecialty clinic appointments at the $\mathrm{BCCH}$. The adolescents and caregivers completed individual, parallel online questionnaires asking if they had a FP and where they would seek medical care for specific health issues (Additional file 1 and Additional file 2). Information on demographics (age, gender, geographic region), number of hospitalizations and number of specialists involved was also collected. We defined a subset population as medically complex if two or more subspecialty clinics were involved in the adolescent's care, and/or the adolescent had at least 1 hospital admission in the past 12 months. The questionnaire asked participants about their relationship with their FP with respect to patient/provider communication, communication between providers, provider knowledge and trust. Concept domains to assess the FP's involvement in care included the perceived role 
of the FP in routine care, the value of the FP's role in context of multiple providers, and access to an FP. The questionnaire was developed by a team of FPs, pediatric/ adult specialists, and the Transition Steering Committee of the Shared Care Youth Transition project [8]. The questionnaire was at a grade 6 reading level and took about 15 min to complete.

For Phase 2, we recruited a further convenience sample $(n=21)$ of adolescent inpatients/ clinic attendees and their caregivers who identified as having a FP. In addition to completing the questionnaire from Phase 1, we interviewed these adolescents and caregiver attendees about their experiences with their FP. The individual responses were recorded in the transcriptions.

The interview questions focused on adolescents' and caregivers' perspectives of communication and role of the FP in their care (Additional file 3). The interviews were $30 \mathrm{~min}$ long on average and were audiotaped and transcribed. Analysis was done using a qualitative description approach [22]. We used constant comparative analysis to derive thematic patterns within the interviews [23]. Coded themes were then independently reviewed and analyzed by two members of the research team who then discussed the themes to reach consensus.

\section{Inclusion and exclusion criteria}

English speaking adolescents 14-18 years of age with a $\mathrm{CHC} / \mathrm{D}$ who required transition to specialist adult care were included, with their caregivers if present. Caregivers who attended with adolescents with cognitive impairment were included even though the adolescent patient could not directly participate in the study. In phase 2, adolescents and their caregivers were excluded if they did not have a FP.

We compared the data sets from the parallel questionnaires completed independently by the adolescent patients and their caregivers and determined there was little variance between the data. However, the caregiver responses were more complete compared to the adolescent responses where questions were often left unanswered. In these cases, we determined that the caregiver represented the most competent coordinator of care and used this single data set of participants for analysis. When adolescents attended the clinic independently or when there were caregiver language barriers, the adolescent data was used for analysis. During the interviews in Phase 2, adolescents contributed equally so therefore both adolescent and caregiver responses were used for qualitative analysis. Data collected from adolescents and their caregivers in phase 1 was excluded from analysis if they indicated they did not have a FP.

\section{Results}

In Phase 1, 84 adolescent-caregiver pairs were recruited between June-August 2014 with a response rate of $90 \%$.
Three pairs indicated not having a FP and were unable to answer questions surrounding access to and relationship with an FP. These pairs were excluded from further data analysis resulting in $n=81$ for phase 1 . In Phase 2, 21 pairs were recruited between June-August 2015 with a response rate of $67 \%$. The main reason for refusing to participate in the study was lack of time. Data was collected from adolescent-caregiver pairs because most visits were attended by an adolescent patient with their caregiver. However, there were four adolescents with cognitive impairment who were not able to participate, and there were ten adolescents whose caregivers did not complete the questionnaire (either not present or language barriers). A total of 102 questionnaires were analyzed representing the most responsible coordinator of care from each pair (92 caregivers and 10 adolescents). In phase 2, 17 caregiver and 19 adolescent interview responses were subjected to qualitative analysis (4 caregivers and 2 adolescents could not complete the interview due to exclusion criteria).

\section{Demographics}

The adolescents were 14-18 years old (mean 16.4 years) and $49 \%(n=50)$ were $16-17$ years old. Forty four percent $(n=45)$ of adolescent patients were male and $56 \%$ $(n=57)$ were female. Eighty three percent $(n=85)$ were from urban centers (within a $1 \mathrm{~h}$ drive of a regional hospital) (Additional file 4).

\section{Access to family physicians}

In Phase $1,96 \%(n=81)$ of adolescents with $\mathrm{CHC} / \mathrm{D}$ reported having a FP. These adolescents were asked if they ever see their FP independently. Only 3.75\% $(n=3)$ indicated they always saw their FP alone. Sixty three percent ( $n=51$ ) always saw their FP accompanied by a parent/ caregiver, while $28 \%(n=23)$ occasionally saw their FP independently. Thirty eight percent $(n=39)$ had seen their FP two times or less over the past 2 years and $34 \%$ $(n=34)$ had not seen their FP in the last 6 months. A further $14 \%(n=14)$ had not seen their FP in 12 months, and for $5 \%(n=5)$, it was over 18 months since the last primary care visit.

\section{Accessing other medical services}

FPs, hospital specialists, internet, alternative health providers, emergency room, and walk-in clinics were accessed by adolescent study participants in the past 12 months (Additional file 4). Most participants identified that they would see their FP exclusively for primary care issues such as immunizations $(74 \%, n=75)$, cold/ flu like symptoms $(68 \%, n=69)$, injury $(69 \%, n=70)$, referrals $(61 \%, n=62)$, completion of forms $(59 \%, n=60)$. However, they were more likely to access specialists for prescription refills $(50 \%, n=51)$. While the FP was the 
sole source of care for $58 \%(n=59)$ of adolescent patients for sexual health, and for $48 \%(n=49)$ of adolescent patients for mental health, a substantial number would seek care from both FPs and specialists for sexual health $(16 \%, n=16)$ and mental health $(29 \%, n=30)$. Fifteen percent $(n=15)$ identified they would exclusively access the subspecialty clinic for mental health and 10\% $(n=10)$ for sexual health. Regarding education about their medical condition, 36\% $(n=37)$ of adolescent patients reported they would seek information from both FP and specialist, 29\% $(\mathrm{n}=30)$ from their specialist alone and 21\% $(n=21)$ from their FP alone (Table 1$)$.

Participants noted they would access other care services in addition or instead of consulting their FP or specialist (Table 2). Other services included the emergency room, walk-in clinics, alternative medicine, and the internet. The internet was selected as the primary source for education about their medical condition $(31 \%, n=$ $32)$, sexual health $(17 \%, n=17)$ and mental health $(17 \%$, $\mathrm{n}=17$ ), while walk-in clinics were preferred for injuries $(18 \%, n=18)$, cold/flu like symptoms $(14 \%, n=14)$ and medication refills $(10 \%, n=10)$. In the interviews, five participants acknowledged that they obtained most of their care from walk-in clinics as their FP was not readily available, too far away, or too difficult to access. As per one caregiver: "Anything outside of his medical condition here, we would go to a walk-in clinic."

\section{Complex patients}

Fifty two percent $(n=53)$ of adolescents were categorized as complex based on having 2 or more subspecialty clinics involved in their care and/or at least 1 hospital admission in the past 12 months. Compared to those who were less complex, complex adolescent patients seemed more likely to see their hospital specialist rather than their FP for sexual health (16\% vs. $10 \%)$ and for mental health issues ( $24 \%$ vs. $14 \%$ ). In the interviews, 4 adolescent/caregiver pairs reported that the majority of their care was with a specialist because the main concern was the significant health issue. As one pair explained, the main health concerns were "above and beyond our family doctor's capabilities" such that "the family doctor could not handle it."

\section{Relationship with FPs}

The majority $(59 \%, n=60)$ of adolescents had the same FP for over ten years (Additional file 4). Most adolescent/caregiver participants shared the same family doctor $(95 \%, n=20)$, and agreed that their communication was generally good to excellent. They depicted communication as being conducive to sharing information (according to one adolescent, "you could talk to him about anything really") and FPs as personable (a caregiver said, "he jokes with him, which is good. It's good to make that connection.") However, the interview data also revealed communication difficulties (Table 3). One adolescent noted his FP was "pretty narrow minded sometimes" and another a caregiver reported, "sometimes we feel like they are just not listening."

When asked to reflect in Phase 2 on the role of the FP as compared to the role of the specialists, most participants $(n=15)$ felt more comfortable with the FP and had a sense that this was their medical home, even though they indicated the FP was "not for specific health problems or questions." They explained this by noting, "he is closer," "we see him more often" and "I've known him longer." For some participants, this sense of continuity and familiarity was a valued attribute. One adolescent explained, "sometimes I'll see him in public and I'll say hi, so definitely family doctor for me." In contrast, 4 participants indicated feeling more comfortable with specialists as they were more knowledgeable and had made a major difference to their care. In one family, the caregiver preferred the care of the specialist while the adolescent preferred his family doctor.

Table 1 Physician services participants would access for specific care needs

\begin{tabular}{|c|c|c|c|c|c|c|c|c|}
\hline \multirow{2}{*}{$\begin{array}{l}\text { Care needs } \\
\text { Total } n=102\end{array}$} & \multicolumn{2}{|c|}{ FP Only } & \multicolumn{2}{|c|}{ BCCH subspecialty clinic only } & \multicolumn{2}{|c|}{ Both } & \multicolumn{2}{|c|}{ Neither } \\
\hline & $\%$ & $n$ & $\%$ & $n$ & $\%$ & $n$ & $\%$ & $\mathrm{n}$ \\
\hline Medication Refill & 40 & 41 & 25 & 26 & 25 & 25 & 10 & 10 \\
\hline Medication Side Effect & 39 & 40 & 17 & 17 & 22 & 22 & 23 & 23 \\
\hline Education about medical condition & 21 & 21 & 29 & 30 & 36 & 37 & 14 & 14 \\
\hline Sexual Health & 58 & 59 & 10 & 10 & 6 & 6 & 26 & 27 \\
\hline Mental Health & 48 & 49 & 15 & 15 & 14 & 14 & 24 & 24 \\
\hline Forms & 59 & 60 & 10 & 10 & 15 & 15 & 17 & 17 \\
\hline Referral & 61 & 62 & 8 & 8 & 24 & 24 & 8 & 8 \\
\hline Injury & 69 & 70 & 0 & 0 & 11 & 11 & 21 & 21 \\
\hline Cold/flu like symptoms & 68 & 69 & 3 & 3 & 10 & 10 & 20 & 20 \\
\hline Immunizations & 74 & 75 & 0 & 0 & 6 & 6 & 21 & 21 \\
\hline
\end{tabular}


Table $\mathbf{2}$ Other services participants would access for specific care needs

\begin{tabular}{|c|c|c|c|c|c|c|c|c|c|c|}
\hline \multirow{2}{*}{$\frac{\text { Care needs }}{\text { Total } n=102}$} & \multicolumn{2}{|c|}{ Emergency Room } & \multicolumn{2}{|c|}{ Walk in Clinic } & \multicolumn{2}{|c|}{ Alternative Medicine } & \multicolumn{2}{|c|}{ Internet } & \multicolumn{2}{|c|}{ Other } \\
\hline & $\%$ & $n$ & $\%$ & $n$ & $\%$ & $n$ & $\%$ & $\mathrm{n}$ & $\%$ & $\mathrm{n}$ \\
\hline Medication Refill & 6 & 6 & 10 & 10 & 2 & 2 & 0 & 0 & 4 & 4 \\
\hline Medication Side Effect & 15 & 15 & 9 & 9 & 2 & 2 & 9 & 9 & 7 & 7 \\
\hline Education about medical condition & 2 & 2 & 2 & 2 & 4 & 4 & 31 & 32 & 4 & 4 \\
\hline Sexual Health & 1 & 1 & 2 & 2 & 4 & 4 & 17 & 17 & 10 & 10 \\
\hline Mental Health & 5 & 5 & 3 & 3 & 4 & 4 & 17 & 17 & 12 & 12 \\
\hline Forms & 0 & 0 & 3 & 3 & 0 & 0 & 5 & 5 & 4 & 4 \\
\hline Referral & 3 & 3 & 7 & 7 & 1 & 1 & 1 & 1 & 3 & 3 \\
\hline Injury & 36 & 37 & 18 & 18 & 3 & 3 & 2 & 2 & 3 & 3 \\
\hline Cold/flu like symptoms & 6 & 6 & 14 & 14 & 4 & 4 & 6 & 6 & 4 & 4 \\
\hline Immunizations & 1 & 1 & 8 & 8 & 2 & 2 & 1 & 1 & 13 & 13 \\
\hline
\end{tabular}

\section{Communication}

With respect to communication between their specialist and FP care providers, most participants felt comfortable that necessary documentation had been sent and received. In their experience, the appropriate lab results and reports had been available in their files "most of the time." However, few had a clear understanding of whether or not this involved any direct verbal communication. Their responses were characterized by vague impressions such as "I believe they would," "I could imagine," or "I'm not really sure." One adolescent expressed, "I'm not a psychic. I don't know what they say".

\section{Perceived knowledge of FP}

When asked about the relative degree to which their health care providers were knowledgeable, most thought that while specialists and FPs had particular skillsets, the specialist possessed considerably more knowledge about their specific condition. One adolescent noted, "If the family doctor was knowledgeable enough I wouldn't be here at this hospital," and one caregiver explained, "I am most comfortable bypassing my family doctor and talking to the specialists."

\section{Care coordination}

The perspective of most of participants was that care coordination was primarily a matter of referral to specialists, and most $(n=14)$ felt their FP was doing this well. As one account illustrated, the participant felt the FP would not be able to fill out medical forms, but could arrange referrals, and acknowledged, "The parts he can do, he will do well." However, in two cases, specialist care was coordinated independently of the FP. In another, the adolescent felt the FP was coordinating care, but the caregiver described herself as coordinating with the specialist. Thus the idea of care coordination did not seem well understood by these families.

When explicitly questioned about their trust of the FP to manage their care, most of the participants $(n=16)$ agreed that their FP was trustworthy. They expressed this as "she knows the family dynamic," "she knows how I react to things," "he would tell us everything," or "she's open." However, for the remainder, there were notable hesitations, including concerns about the level of knowledge. One adolescent stated, "He isn't helpful, particularly when I am in pain."

In Phase 2, all but one participant felt that having an FP had improved their overall health, explaining this as having to do with the continuity of care and breadth of interest in factors affecting their health. "She knows him, found his problem to start with, and has medical records," "she is mostly the reason I can get better," and noting that, when seeing a specialist "all the other stuff

Table 3 Adolescents and caregivers' relationship with FP

\begin{tabular}{|c|c|c|c|c|c|c|c|c|}
\hline \multirow{2}{*}{$\frac{\text { Questions regarding relationship with FP }}{\text { Total } n=102}$} & \multicolumn{2}{|c|}{ Not at all } & \multicolumn{2}{|c|}{ Slightly } & \multicolumn{2}{|c|}{ Very } & \multicolumn{2}{|c|}{ Extremely } \\
\hline & $\%$ & $\mathrm{n}$ & $\%$ & $\mathrm{n}$ & $\%$ & $\mathrm{n}$ & $\%$ & $\mathrm{n}$ \\
\hline How comfortable do you and your youth feel asking questions of their FP & 5 & 5 & 45 & 46 & 42 & 43 & 7 & 7 \\
\hline How likely are you and your youth to recommend your youth's FP & 9 & 9 & 23 & 23 & 43 & 44 & 25 & 25 \\
\hline How knowledgeable do you and your youth feel your youth's FP is about their medical condition & 8 & 8 & 45 & 46 & 38 & 39 & 8 & 8 \\
\hline How helpful is your FP at explaining your youth's medical condition & 13 & 13 & 35 & 36 & 37 & 38 & 14 & 14 \\
\hline How easy is it for your youth to get to their FP's office & 11 & 11 & 26 & 27 & 41 & 42 & 21 & 21 \\
\hline How easy is it to schedule urgent appointments with your youth's FP when your youth is ill & 20 & 20 & 34 & 35 & 32 & 33 & 13 & 13 \\
\hline
\end{tabular}


doesn't get talked about." Many aspects of care were more accessible with a FP than a specialist.

\section{Discussion}

As most of the adolescent patients arrived at subspecialty appointments with their caregiver and did not see the specialist alone during the clinic visit, we determined that during the transition-planning period (i.e. prior to transfer to adult care), caregivers were particularly influential in determining where and whom they sought for care. However, during the interviews every adolescent could describe their relationship with their FP, giving their impressions regarding care management and the role of their physicians. This finding suggests that with appropriate support and encouragement, adolescent patients could be involved in developing independent decision making for their own health care, an important component of self-management.

Most participants identified a long-term relationship with a FP, listing continuity, familiarity and proximity to be valued attributes of their FP. Overall, adolescent patients and caregivers appreciated the contributions their FPs could make and recognized the distinction between the skillset and focus of the FP and the specialist. The majority had a positive relationship with the FP, feeling they were trustworthy and improved the adolescent's overall health. Communications with their FP and between specialists and FPs were generally perceived as good, although few fully understood the intended alignment between the generalist and specialist systems. Several felt the FP's role of coordinating medical care was primarily a matter of referral to specialists.

Despite the positive recognition of the role of the FP, there was a concerning lack of regular involvement of the FP in the adolescent's medical care as determined by the number of reported patient visits in the previous two years. The most cited reason was that the FP was unavailable, too far, or too difficult to access, resulting in some participants choosing walk-in clinics for primary care. With increasing waiting times and difficulty in getting an appointment, patients are often turning to walk-in clinics. However, recently recommended practice management strategies designed to increase efficacy in the medical home model may mitigate this issue [24].

In some cases participants identified specialists as their source for primary health care, particularly when the adolescent patients had medically complex conditions (53\% of our sample). These participants seem most likely to view their specialist as the exclusive care provider for concerns such as mental and sexual health. Some participants felt that the adolescent patient's medical conditions were beyond the FP's capabilities and therefore went directly to the specialist for medical concerns, establishing their medical home at the subspecialty clinic rather than with their FP. Not only is this an ineffective use of health care resources, it creates missed opportunities for establishing the FP's office as the adolescent's medical home [14]. When the specialist provides primary health care it creates a more significant handover at the time of transfer to adult specialist services. The FP who is unfamiliar with the patient needs to rapidly acquire the knowledge of a complex, chronic condition and develop a new patient/provider relationship to assume the role as the most responsible physician. Transfer to an entirely new set of providers is more likely to exacerbate the fear and resistance to transfer [25]. This is a significant concern as such adolescents are at higher risk for medical complications if lost to follow up care after transfer [5].

Given the paucity of evidence-based literature to guide practice or policy surrounding the role of the primary health provider during transition to adult services, there are some limitations in this novel study. The study was based on a small sample size, especially when narrowed down to more complex patients. A larger sample size would benefit future investigations to determine if there is a difference in the attachment to the FP between complex and non-complex adolescents, and where they establish their medical home prior to transition. Furthermore, participants were recruited via a convenience sample from multiple clinics and therefore our sample was not randomized. The data used for analysis represented the most competent coordinator of care, which was the caregiver in most cases except when the adolescent attended the clinic alone or when there were caregiver language barriers. Some questions in the youth questionnaires were left unanswered, and this may have been due to lack of knowledge or engagement with the questionnaire. Obtaining a complete and reliable set of adolescent responses for analysis would be valuable in highlighting the adolescents' use of the internet, walk-in clinics and youth clinics for medical care, particularly for sensitive topics such as mental and sexual health. Using a shorter, simplified, youth-friendly questionnaire with images designed to engage adolescents could yield such results in future studies.

\section{Conclusions}

There is limited empiric evidence to guide primary care interventions to improve transition outcomes for adolescents with $\mathrm{CHC} / \mathrm{D}$ 's [1]. As a result of this study, we believe that opportunities exist for strengthening the attachment adolescents with $\mathrm{CHC} / \mathrm{Ds}$ have with their FP as the primary manager of care. As the FP becomes the unequivocal most responsible physician after transfer, facilitating a strong FP-patient relationship and establishing the medical home results in a more effective transition $[12,13]$. Given that only $3.75 \%$ of adolescents 
with $\mathrm{CHC} / \mathrm{D}$ 's regularly see their FP alone, health care providers and caregivers should encourage adolescents to become more independent in the management of their own health care. Preparation for transition should start early in childhood [26] so that they experience a graded responsibility that facilitates their interactions with the health care system separate from that of their caregivers.

Responsibility for improving adolescent patient/caregiver health literacy and helping them understand the role of the FP in the adolescent's health management should be shared between FPs and pediatric specialists. In order to ensure continuity of care past the pediatric age, specialists have an opportunity to facilitate the relationship with their FP by encouraging adolescents to see their FP for primary health issues in between specialist visits. With support from pediatric specialists, including clear communication as to the needs of these special populations, FPs can play an integral role in improving health outcomes for adolescents with $\mathrm{CHC} / \mathrm{Ds}$ during transition.

\section{Additional files}

Additional file 1: Caregiver Questionnaire Questionnaire completed by caregivers of adolescent patients. (DOCX $92 \mathrm{~kb}$ )

Additional file 2: Youth Questionnaire Questionnaire completed by adolescent patients. (DOCX $92 \mathrm{~kb}$ )

Additional file 3: Extension interview questions These interview questions focused on adolescents' and caregivers' perspectives of communication and role of the FP in their care. (DOCX 77 kb)

Additional file 4: Appendix This appendix includes supplemental information regarding patient demographics, access to medical services, and relationship with the FP. (DOCX $47 \mathrm{~kb}$ )

\section{Abbreviations}

CHC/D: Chronic health condition and/or disability; FP: Family physician

\section{Acknowledgements}

We thank Mary Paone as well as the On TRAC team for their involvement and support throughout this project.

\section{Funding}

Funding was provided by the Child and Family Research Institute. The CFRI funds were used for gift cards for study participants in addition to summer research student salary to support collection, analysis, and interpretation of data.

\section{Availability of data and materials}

Raw data was generated at the British Columbia Children's Hospital in Vancouver, British Columbia, Canada. The data sets used and analyzed during the current study are available from the corresponding author on reasonable request.

\section{Authors' contributions}

AH collected the data in phase one of the study, interpreted the data and contributed in writing the manuscript. SW initiated the conception and design of the study, played a role in data interpretation and writing the manuscript, and oversaw the project. STsai collected the data in phase two of the study, contributed to data analysis and editing of the manuscript. SH analyzed, organized and computed the overall data collected, as well as editing the manuscript. SThorne analyzed and interpreted the qualitative data and contributed in writing and editing the manuscript. All authors read and approved the final manuscript.

\section{Ethics approval and consent to participate}

The University of British Columbia Research Ethics Board provided ethics approval for the study and informed consent to participate was obtained from all participants. The need for parental consent was waived by the research ethics board for adolescents 14-15 years old as they were deemed mature minors and therefore competent to provide consent to participate in the study.

\section{Consent for publication}

Not applicable.

\section{Competing interests}

The authors declare that they have no competing interests.

\section{Publisher's Note}

Springer Nature remains neutral with regard to jurisdictional claims in published maps and institutional affiliations.

\section{Author details}

${ }^{1}$ University of Alberta, 116 St \& 85 Ave, Edmonton, AB T6G 2R3, Canada. ${ }^{2}$ University of British Columbia, 2329 West Mall, Vancouver, BC V6T 1Z4, Canada. ${ }^{3}$ Child Family Research Institute, 950 W 28th Ave, Vancouver, BC V5Z 4H4, Canada. ${ }^{4}$ University of British Columbia School of Nursing, T201-2211 Wesbrook Mall, Vancouver, BC V6T 2B5, Canada.

Received: 28 June 2017 Accepted: 14 August 2018

Published online: 23 August 2018

\section{References}

1. Bhawra JTA, Cohen E, Moore Hepburn C, Guttmann A. Primary care interventions to improve transition of youth with chronic health conditions from paediatric to adult healthcare: a systematic review. BMJ Open. 2016; 6(5):e011871. https://doi.org/10.1136/ bmjopen-2016-71.

2. Zhou H, Roberts P, Dhaliwal S, Della P. Transitioning adolescent and young adults with chronic diseases and/or disabilities from pediatric to adult care services: an integrative review. J Clin Nurs. 2016;25(21-22):3113-30. https:// doi.org/10.1111/jocn.13326.

3. Harris MA, Freeman KA, Duke DC. Transitioning from pediatric to adult health care: dropping off the face of the earth. Am J Lifestyle Med. 2011; 5(1):85-91.

4. Reid GJ, Irvine MJ, McCrindle BW, Sananes R, Ritvo PG, Siu SC, et al. Prevalence and correlates of successful transfer from pediatric to adult health care among a cohort of young adults with complex congenital heart defects. Pediatrics. 2004;113:e197-205.

5. Prestidge C, Romann A, Djurdjev O, Matsuda-Abedini M. Utility and cost of a renal transplant transition clinic. Pediatr Nephrol. 2012;27(2):295-302.

6. American Academy of Pediatrics, American Academy of family physicians, American College of Physicians, transitions clinical report authoring group, Cooley WC, Sagerman PJ. Supporting the health care transition from adolescence to adulthood in the medical home. Pediatrics. 2011;128(1):182200.

7. McManus MA, White P, Barbour A, Downing B, Hawkins K, Quion N, et al. Pediatric to adult transition: a quality improvement model for primary care. J Adolesc Health. 2015;56(1):73-8. https://doi.org/10.1016/j.jadohealth.2014. 08.006 .

8. Whitehouse SR. When chronic care youth age out: transition planning. BCMJ. 2015;57(2):70-1.

9. Mennito SH, Clark JK. Transition medicine: a review of current theory and practice. South Med J. 2010;103:339-42.

10. Robertson $L$. When should young people with chronic rheumatic disease move from paediatric to adult-centred care? Best Pract Res Clin Rheumatol. 2006;20:387-97.

11. Reiss JG, Gibson RW, Walker LR. Health care transition: youth, family, and provider perspectives. Pediatrics. 2005;115(1):112-20.

12. Lotstein DS, McPherson M, Strickland B, Newacheck PW. Transition planning for youth with special health care needs: Results from National Survey of children with special health care needs. Pediatrics. 2005;115(6):1562-8. 
13. McManus MA, Pollack LR, Cooley WC, McAllister JW, Lotstein D, Strickland B, et al. Current status of transition preparation among youth with special needs in the United States. Pediatrics. 2013;131(6):1090-7. https://doi.org/10. 1542/peds.2012-3050.

14. College of Family Physicians of Canada. A vision for Canada: Fam Pract - the patient's medical home, 2011. http://www.cfpc.ca/uploadedFiles/Resources/ Resource_Items/PMH_A_Vision_for_Canada.pdf Accessed 17 May, 2016.

15. Amed S, Nuernberger K, McCrea P, Reimer K, Krueger H, Aydede SK, et al. Adherence to clinical practice guidelines in the management of children, youth, and young adults with type 1 diabetes: a prospective population cohort study. J Pediatr. 2013;163(2):543-8.

16. DeBaun MR, Telfair J. Transition and sickle cell disease. Pediatrics. 2012; 130(5):926-35. https://doi.org/10.1542/peds.2011-3049.

17. Shaw TM, Delaet DE. Transition of adolescents to young adulthood for vulnerable populations. Pediatr Rev. 2010;31(12):497-504. https://doi.org/10. 1542/pir.31-12-497.

18. Tucker LB, Cabral DA. Transition of the adolescent patient with rheumatic disease: issues to consider. Rheum Dis Clin N Am. 2007;33:661-72. https:// doi.org/10.1016/j.rdc.2007.07.005.

19. Damiano PC, Momany ET, Tyler MC, Penziner AJ, Lobas JG. Cost of outpatient medical care for children and youth with special health care needs: investigating the impact of the medical home. Pediatrics. 2006; 119(6):1187-94.

20. American Academy of Pediatrics. The medical home: medical home initiatives for children with special needs project advisory committee. Pediatrics. 2002:110(1):184-6.

21. Young RA, Marshall SK, Domene JF, Graham M, Logan C, Zaidman-Zait A, et al. Transition to adulthood as a parent-youth project: governance transfer, career promotion, and relational processes. J Couns Psych. 2008; 55(3):297-307.

22. Sandelowski M. Whatever happened to qualitative description? Res Nurs Health. 2000;23:334-40.

23. Thorne S. Interpretive description: qualitative research for applied practice. New York: Routledge; 2016

24. Murray MM, Tantau C. Same day appointments: exploding the access paradigm. Fam Pract Manag. 2000;7(8):45-50.

25. Tuchman LK, Slap GB, Britto MT. Transition to adult care: experiences and expectations of adolescents with a chronic illness. Chind Care Health Dev. 2008:34(5):557-63.

26. Kaufman M, Pinzon J. Transition to adult care for youth with special health care needs. Position Statement, Canadian Paediatric Society. Paediatr Child Health. 2007:12:785-88.

Ready to submit your research? Choose BMC and benefit from:

- fast, convenient online submission

- thorough peer review by experienced researchers in your field

- rapid publication on acceptance

- support for research data, including large and complex data types

- gold Open Access which fosters wider collaboration and increased citations

- maximum visibility for your research: over $100 \mathrm{M}$ website views per year

At $\mathrm{BMC}$, research is always in progress.

Learn more biomedcentral.com/submissions 